\title{
Encapsulated Agents of Hybrid Order Discrete Dynamics
}

\author{
Yunzhong Song ${ }^{\dagger}$ \\ School of Electrical Engineering and Automation, Henan Polytechnic University, 2001 Century Avenue \\ Jiaozuo, 454003, P.R.China
}

Weicun Zhang

School of Automation and Electrical Engineering, University of Science and Technology Beijing, 30Xueyuan Road Beijing, 100083, P.R. China

Fengzhi Dai

School of Electronic Information and Automation, Tianjin University of Science and Technology, 1038 Dagu Nanlu Tianjin, 300222, P.R. China

Huimin Xiao

School of Computer and Information Engineering, Henan University of Economics and Law, 180 Jinshui Donglu Zhengzhou, 450046, P.R.China

\author{
Shumin Fei \\ School of Automation, South East University, 2 Sipai Lou \\ Nanjing, 210096, P.R.China
}

E-mail:songhpu@126.com,weichunzhang@263.net,daifz@tust.edu.cn,xiaohm@huel.edu.cn,smfei@seu.edu.cn

\begin{abstract}
This paper is targeting to investigate the encapsulated cell realization of the hybrid order agents in discrete time, where agents are composed by two different dynamic order ones, one is the first order and the other is the second order, the work is the counter of its continuous ones of the encapsulated agents. To be further, the first order agents are assigned at low speed, and the second order agents are selected at comparatively higher speed. To overcome the obstacle brought by difference of the sampling rates, the lifting technology is taken into use to analyze the encapsulated system. Theoretical analysis and simulation results are made available for the further reference.
\end{abstract}

Keywords: encapsulated agents, hybrid order, discrete dynamics, lifting techniques, consensus

\section{Introduction}

Multi-agent systems have been extensively explored these days for their theoretical values and potential prospects in real engineering applications ${ }^{1-9}$. Among them, the heterogeneous agents were paid much more attention, partially because of their widely distributed in industries, social activities and biological groups; one of the striking point of heterogeneities of the multi-agents is their difference in dynamics, especially of dynamic orders. The hybrid order dynamics of multi-agents were firstly put forward and solved by match principle or

${ }^{\dagger}$ Corresponding author

(C) The 2021 International Conference on Artificial Life and Robotics (ICAROB2021), January 21 to 24, 2021 
match equivalence, i.e. protocols should be different for agents with different dynamic orders ${ }^{3,6}$.

However, it is not so wise for us just to stop at the low level of the multi-agents, something new should be added to push this research further. Is it possible to pack the agents with hybrid order together? Does that bring us much more promising solutions to the real engineering problems? These problems are full of the meaning. One of the work of this direction has been done, it is for hybrid order agents packed in an encapsulated cell. The dynamics for them is described in continues time ${ }^{3}$. In this note, discrete time agents of the hybrid order packed in an encapsulated cell will be touched upon here.

The following of this note will be arranged as follows: Problem description will be provided at first, then followed by theoretical analysis and simulations, some comments will also be available in this part; the last part will be conclusions. Acknowledgements will be acknowledged for the fund support.

\section{Problem Description}

The dynamics of the encapsulated agents are described in the following equations:

$$
\left\{\begin{array}{l}
X_{j}^{i}(k+1)=f\left(X_{j}^{i}(k), P\right)+U_{j}^{i}(k) \\
Y^{i}(k)=x_{1}^{i}(k)
\end{array}\right.
$$

Here, in Equation (1), index $i$ is used to mark the layer of the agents, and index $j$ is used to mark the order of the agents of the layer $i ; u(k)$ is the controlled input for the agents, it comes from three different places, one is from the agent itself, the other is from the other agents of the same layer, and the third is from the other layer agents; the output signal is extracted from the first order agents; the capital $x$ and $u$ is employed to express the packed state variables, i.e. to the agent systems combined by the first and second order dynamics, both position sub-states and velocity sub-states are covered by the capital $x$, the capital letter $p$ is used to denote the parameters of the agent systems; and the controlled input can be inferred correspondingly.

In case of simplicity and without loss of generality, we assume the multi-agents system is composed by two layers; each layer includes two different agents, among them one is the first order and the other is the second order. So, the Equation (1) can be refined as follows:

$$
\left\{\begin{array}{l}
\frac{x_{1}^{i}(k+1)-x_{1}^{i}(k)}{n_{0} T_{0}}=u_{1}^{i}(k) ; \\
\frac{x_{2}^{i}(k+1)-x_{2}^{i}(k)}{T_{0}}=v_{2}^{i}(k) ; \\
\frac{v_{2}^{i}(k+1)-v_{2}^{i}(k)}{T_{0}}=u_{2}^{i}(k) ; \\
y^{i}(k)=x_{1}^{i}(k) ;
\end{array}\right.
$$

Corresponding to Equation (1),

$$
\left\{\begin{array}{l}
X_{1}^{i}(k)=x_{1}^{i}(k) ; \\
X_{2}^{i}(k)=\left[\begin{array}{l}
x_{2}^{i}(k) \\
v_{2}^{i}(k)
\end{array}\right] ;
\end{array}\right.
$$

As to the controlled inputs, both for the first order agents and the second agents, are enforced by three different parts, one is from the individual agent itself, the another is from the same layer of the different order agents, and the third is from the other layer agents. And in this way, we have system level of the whole agents, and sub-level of the layer, followed by the atom-level agents with different dynamic orders.

Notation 1: Parameters of $n_{0}$ and $T_{0}$ is assigned to represent the times of the sampling period and standard sampling period, respectively; since the first order agent system only has one integrator, and the second order agent system has two integrators, the information updating rate for the second is much more slower than the first agent system; and in this kind of sense, we chose $T_{0}$ as 1 and $\mathrm{n}_{\mathrm{o}}$ as 4 .

\section{Theoretical Analysis and Simulation Results}

This part will contribute for theoretical analysis and simulation results, some comments are also offered to trigger the prospective topics.

\subsection{Theoretical analysis}

Take two homogeneous encapsulated cell agent systems as an example, each one of them is composed of one of the first order agent and one of the second order agent, to be specific, for the first cell encapsulated agent system, it can be expressed as: 


$$
\left\{\begin{array}{l}
x_{1}^{1}(k+1)=x_{1}^{1}(k)+4 u_{1}^{1}(k) ; \\
x_{2}^{1}(k+1)=x_{2}^{1}(k)+v_{2}^{1}(k) ; \\
v_{2}^{1}(k+1)=v_{2}^{1}(k)+u_{2}^{1}(k) ; \\
y^{1}(k)=x_{1}^{1}(k) ;
\end{array}\right.
$$

Correspondingly, the second cell encapsulated agent system can be expressed as:

$$
\left\{\begin{array}{l}
x_{1}^{2}(k+1)=x_{1}^{2}(k)+4 u_{1}^{2}(k) ; \\
x_{2}^{2}(k+1)=x_{2}^{2}(k)+v_{2}^{2}(k) ; \\
v_{2}^{2}(k+1)=v_{2}^{2}(k)+u_{2}^{2}(k) ; \\
y^{2}(k)=x_{1}^{2}(k)
\end{array}\right.
$$

Notation 2: The dynamic of the first order agent in cell encapsulated agent system is only influenced by its own dynamics and the dynamics of the second order agent of the same cell. To be different, the dynamic of the second order agent in cell encapsulated agent system can be altered by its own dynamic and also the dynamic of the first order agent, which is assigned to the same cell, besides that, the output of the other cell agent system can also contribute to the dynamics of the second order agent.

Notation 3: Consider the different sampling speed, the signal of the second order agent feed to the first order agent is the 4 times period delayed signal, that embodies the lifting technique.

For the first cell encapsulated agent system, the controlled input can be denoted as follows:

$$
\left\{\begin{array}{l}
x_{1}^{1}(k+1)=x_{1}^{1}(k)+4 u_{1}^{1}(k) ; \\
u_{1}^{1}(k)=u_{11}^{1}(k)+u_{12}^{1}(k) ; \\
u_{11}^{1}(k)=-\alpha_{1} x_{1}^{1}(k) ; \\
u_{12}^{1}(k)=\beta_{1}\left[x_{2}^{1}(k-4)-x_{1}^{1}(k)\right] ; \\
x_{2}^{1}(k+1)=x_{2}^{1}(k)+v_{2}^{1}(k) ; \\
v_{2}^{1}(k+1)=v_{2}^{1}(k)+u_{2}^{1}(k) ; \\
u_{2}^{1}(k)=u_{21}^{1}(k)+u_{22}^{1}(k)+u_{23}^{1}(k) ; \\
u_{21}^{1}(k)=-\alpha_{2} x_{2}^{1}(k)-\alpha_{3} v_{2}^{1}(k) ; \\
u_{22}^{1}(k)=\beta_{2}\left[x_{1}^{1}(k)-x_{2}^{1}(k-4)\right] ; \\
u_{23}^{1}(k)=\beta_{3}\left[x_{1}^{2}(k)-x_{1}^{1}(k)\right] ; \\
y^{1}(k)=x_{1}^{1}(k) ;
\end{array}\right.
$$

And the second cell encapsulated agent system, the controlled input can be denoted as follows:

$$
\left\{\begin{array}{l}
x_{1}^{2}(k+1)=x_{1}^{2}(k)+4 u_{1}^{2}(k) ; \\
u_{1}^{2}(k)=u_{11}^{2}(k)+u_{12}^{2}(k) ; \\
u_{11}^{2}(k)=-\alpha_{1} x_{1}^{2}(k) ; \\
u_{12}^{2}(k)=\beta_{1}\left[x_{2}^{2}(k-4)-x_{1}^{2}(k)\right] ; \\
x_{2}^{2}(k+1)=x_{2}^{2}(k)+v_{2}^{2}(k) ; \\
v_{2}^{2}(k+1)=v_{2}^{2}(k)+u_{2}^{2}(k) ; \\
u_{2}^{2}(k)=u_{21}^{2}(k)+u_{22}^{2}(k)+u_{23}^{2}(k) ; \\
u_{21}^{2}(k)=-\alpha_{2} x_{2}^{2}(k)-\alpha_{3} v_{2}^{2}(k) ; \\
u_{22}^{2}(k)=\beta_{2}\left[x_{1}^{2}(k)-x_{2}^{2}(k-4)\right] ; \\
u_{23}^{2}(k)=\beta_{3}\left[x_{1}^{1}(k)-x_{1}^{2}(k)\right] ; \\
y^{2}(k)=x_{1}^{2}(k) ;
\end{array}\right.
$$

Parameters of the whole system can be listed as follows:

$$
\left\{\begin{array}{l}
\alpha_{1}=\frac{1}{8} ; \alpha_{2}=\frac{1}{2} ; \alpha_{3}=\frac{5}{3} \\
\beta_{1}=\frac{1}{8} ; \beta_{2}=\frac{1}{2} ; \beta_{3}=\frac{1}{16}
\end{array}\right.
$$

Notation 4: The parameters are selected to guarantee the stability of the whole system.

\subsection{Simulation results}

When the initial values are provided, under the simulation environment like SIMULINK, the Simulink results are easily to be made possible. Figure 1 is the simulation results of the encapsulated system:

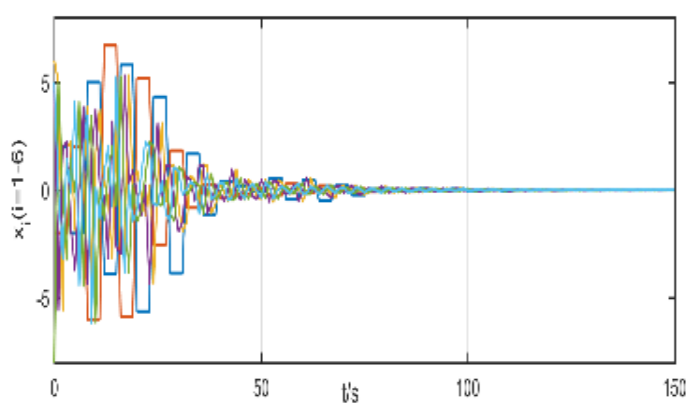

Fig.1 State curves of the system 
From the simulation results, it could be seen clearly that all of the states of the system are driven to the stable equilibrium.

Notation 5: The different equilibrium values can be made possible if we assign different working modes of the sub-system.

\subsection{Some comments}

In order to make the work tight and complete, two comments are offered here.

Comment 1: Agent system is kicked off by studying the same order ones, especially of the first order agents and the second order agents, after then the hybrid order agent systems are initiated ${ }^{3,6}$ and references there in followed by colleagues of the control field. Is it possible for us to come back to the same agents but with much more higher viewpoints?

Comment 2: Encapsulated cell agent systems are the right way for us to dig deep on the road of the same at low level, the different to deepen of the same, and come to the same in a higher level.

\section{Conclusion}

This note contributes to the background introduction, theoretical analysis and simulation research of the encapsulated cell agents with discrete dynamics. The value of this note is obvious in several aspects:

(1) It reiterates the importance of the layer of the system, now it is clear that the encapsulated cell agents are divided into three different levels, from the top to the down, they are the system level, the cell level, and the agent level;

(2) According to our knowledge, it is first discussion about the sampling speed of the hybrid order agents, lifting techniques are borrowed to design the controller;

(3) For the complex engineering system, the sub-systems are always intertwined with each other, hierarchy should be considered of course, and in this sense, our work is meaningful;

Besides, the complexity is the essential characteristics, time-delay, coupling, fragility, robust property et al, all of them should be taken into count ${ }^{10-12}$, it is a long way for us to explore.

\section{Acknowledgements}

This work is partially supported by NSFC Grant (61340041 and 61374079) and the Project-sponsored by
SRF for ROCS, SEM to Yunzhong Song as well as Natural Science Fund of Henan Province (182300410112).

\section{References}

1. R. Olfati, R. Murray, Consensus problems in networks of agents with switching topology and time delays, IEEE Transactions on Automatic Control, 49(2004) 1520-1533.

2. Y.Song, F. Dai, H. Xiao and S. Fei, No free lunch principle in agent swarm systems: one Case Study, Journal of Advances in Artificial Life Robotics, 1 (2020) 33-37.

3. Y.Song, F. Dai, H. Xiao, On the complexity of encapsulated agent cell, Proceedings of 2019 Chinese Intelligent Systems Conference, (2019) 24-30

4. Y. Song, Z. Fu, F. Wang, Socialized multi-agent system rendezvous via networks of networks, Journal of Robotics, Networking and Artificial Life, 2 (2016) 136-139.

5. Y. Song, Z. Fu, F. Wang, Flock guiding of hybrid agents via root block, Journal of Robotics, Networking and Artificial Life, 5 (2019) 245-248

6. Y. Song, Consensus of agents with mixed linear discrete dynamics, International Journal of Control, Automation and Systems, 14 (2016) 1139-1143.

7. Y. Song, W. Zhao, Multi-agent system rendezvous via refined social system and individual roles, WSEAS Transactions on Systems and Control, 9(2014),526-532

8. Y. Hong, J. Hu, L. Gao, Tracking control for multi-agent consensus with an active leader and variable topology, Automatica, 42(2006) 1177-1182.

9. Y. Hong, G. Chen, L. Bushenell, Distributed observers design for leader-following control of multi-agent networks, Automatica, 44(2008) 846-850.

10. Y. Jia, Robust control with decoupling performance for steering and traction of $4 \mathrm{WS}$ vehicles under velocityvarying motion, IEEE Transactions on Control Systems Technology, 8(2000) 554-569.

11. Y. Jia, Alternative proofs for improved LMI representations for the analysis and the design of continuous-time systems with polytopic type uncertainty: a predictive approach, IEEE Transactions on Automatic Control, 48(2003) 14131416

12. Y. Jia, General solution to diagonal model matching control of multi-output-delay systems and its applications in adaptive scheme, Progress in Natural Science, 19(2009), 79-90.

(C) The 2021 International Conference on Artificial Life and Robotics (ICAROB2021), January 21 to 24, 2021 